\title{
Sub-Carrier-Multiplexed Duplex 64-QAM Radio-over-Fiber Transmission for Distributed Antennas
}

\author{
Salman Ghafoor and Lajos Hanzo
}

\begin{abstract}
We demonstrate the feasibility of Radio Over Fiber (ROF) transmission of 4 Sub-Carrier Multiplexed (SCM) 64QAM data streams to a pair of low-complexity Radio Access Units (RAUs), which form part of a virtual MIMO architecture under the control of a Central Unit (CU). The ROF signal is transmitted over low frequency RF carriers and heterodyne detection is used at the RAUs. The 4 coherent optical carriers used for data transmission as well as for heterodyning at the RAUs are generated using a single laser rather than several inevitably non-coherent lasers. Our proposed system transmits duplex data of $480 \mathrm{Mbit} / \mathrm{s}$ and $240 \mathrm{Mbit} / \mathrm{s}$ in the downlink and uplink directions, respectively at mm-wave frequencies of $25 \mathrm{GHz}$ or $50 \mathrm{GHz}$.
\end{abstract}

Index Terms-Radio-over-fiber transmission, distributed antennas, 64QAM.

\section{INTRODUCTION}

D ISTRIBUTED Antenna Systems (DAS) using mm-waves and sophisticated, yet low-complexity $6 \mathrm{bit} / \mathrm{symbol} 64$ QAM transmissions are proposed for a novel DAS application. DASs provide a high capacity per subscriber by re-using the same frequency band in all the adjacent cells [1]. In this scenario, the optical fiber backbone linking the Radio Access Units (RAUs) plays an important role as a benefit of its high bandwidth, transparency to RF signals and low attenuation. Hence the RAUs are connected to a Central Unit (CU) by their optical fiber backbone and all the signal processing tasks can be performed at the CU, where the RAU antennas act as elements of a virtual Multiple Input Multiple Output (MIMO) architecture [1], as shown in Fig 1. Consider Link 1, which consists of two RAUs connected through two spans of optical fiber with a CU. The RAUs transmit RF signals at mm-wave to the Mobile Stations (MSs), which are connected to a particular RAU depending upon their received signal quality. Transmission of mm-waves utilizing remote heterodyne detection (RHD) at the RAU by the use of separate lasers has been proposed in [2], [3]. However, the separate laser sources used in [2] and [3] require complex signal processing for achieving coherence amongst them. RHD is achieved by generating multiple coherent sidebands from a single laser in [4] to transmit baseband data at a mm-wave frequency from a single RAU. However, the technique used in [4] transmits a highpower optical carrier along with the sidebands through the

Manuscript received August 24, 2011. The associate editor coordinating the review of this letter and approving it for publication was O. Dobre.

The authors are with the School of ECS, University of Southampton, SO17 1BJ, United Kingdom (e-mail: \{sag1z07, lh\}@ecs.soton.ac.uk; http://wwwmobile.ecs.soton.ac.uk).

The financial support of the National University of Science and Technology (NUST), Pakistan, as well as that of the EPSRC, UK under the auspices of the India-UK Advanced Technology Centre and the China-UK Bridge is gratefully acknowledged.

Digital Object Identifier 10.1109/LCOMM.2011.101711.111794

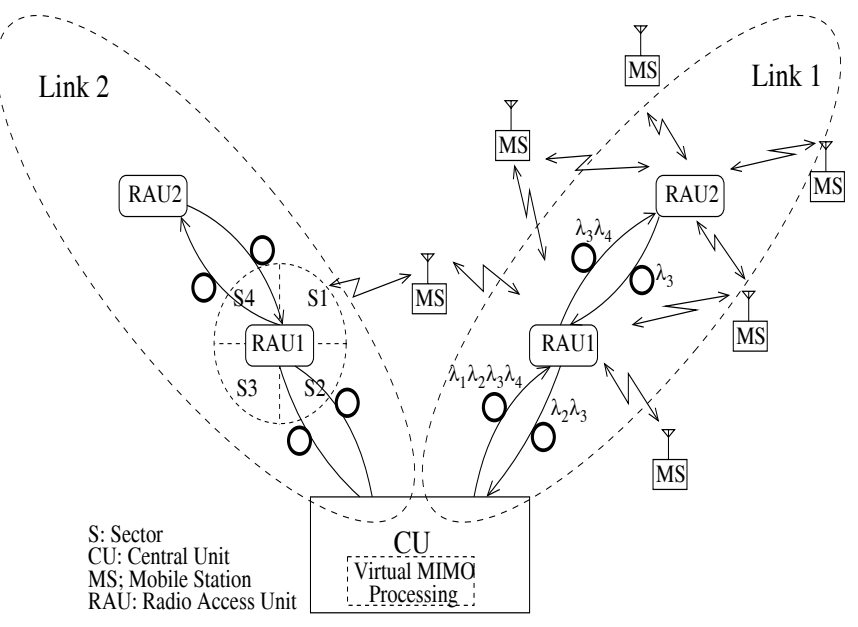

Fig. 1. A Virtual MIMO architecture

fiber, which may distort the vulnerable 6 bit/symbol 64-QAM ROF signal due to Cross Phase Modulation (XPM) which is less of a problem for conventional 1 bit/symbol On-Off Keying (OOK) [5]. The XPM imposes phase noise on the ROF signal, which is converted to amplitude noise by the fiber-induced dispersion [5]. Two different methods of generating multiple coherent sidebands using cascaded optical modulators and/or high frequency local oscillators to generate multiple mm-wave frequencies were described in [6]. Since cascaded modulators are used, the number of components in the system is increased and both methods of [6] transmitted only uni-directional data. Bidirectional data transmission at two mm-wave frequencies to two RAUs using a single modulator has been demonstrated in [7]. However, the use of high-frequency local oscillators and a modulator coupled with narrow-band optical filtering makes the system of [7] less attractive. Furthermore, all the methods mentioned above transmit only 1 bit/symbol. In [8], multi-level modulation formats have been used, but the system requires a high frequency local oscillator and a modulator to transmit unidirectional data and only a single RF channel may be transmitted by a single RAU. Against this background, our novel contributions are:

1- We demonstrate the feasibility of transmitting 4 Sub-Carrier Multiplexed (SCM) 6 bit/symbol 64QAM mm-wave signals in a DAS by incorporating coherent optical carriers in the transmitted signal for heterodyne detection at the RAUs.

2- Our scheme transmits ROF signals simultaneously to two different RAUs in a duplex architecture and at two different mm-wave frequencies, using only a single laser source located at the CU.

The simulation tool used for our performance study is the commercial software VPITransmissionMaker 8.5 
(www.vpiphotonics.com).

\section{The Proposed Virtual MiMO Architecture}

Fig. 2 shows the design of Link 1 seen in Fig 1. Four Continous Wave (CW) optical signals are generated from a single Laser Diode (LD) using the Optical Carrier Suppression (OCS) technique of [9]. The sinusoidal signal applied to the Dual-Drive Mach Zehnder Modulator (DD-MZM) of Fig 2 for the generation of multiple sidebands has a frequency of $12.5 \mathrm{GHz}$ and an amplitude of 0.7 times $V_{\pi}$, where $V_{\pi}$ is the voltage required to induce a phase shift of $\pi$ for each of the DD-MZM arms. A more detailed discussion on the use of DD-MZM for OCS technique is mentioned in [9]. The output of the DD-MZM which is amplified using the Erbium Doped Fiber Amplifier (EDFA) of Fig 2, is fed to a $1 \times 4$ Arrayed Waveguide Grating (AWG) having a channel spacing of $25 \mathrm{GHz}$. Each channel of the AWG performs Gaussian filtering with a $3 \mathrm{~dB}$ bandwidth of $25 \mathrm{GHz}$ centered at the odd-indexed optical side-bands generated during OCS. The first-order odd-indexed side-bands are labelled $\lambda_{2}$ and $\lambda_{3}$, while the second-order odd-indexed side-bands are labelled $\lambda_{1}$ and $\lambda_{4}$, as shown in Fig 2. The first-order side-bands $\lambda_{2}$ and $\lambda_{3}$ have a higher Optical Signal to Noise Ratio (OSNR) compared to the second order side-bands $\lambda_{1}$ and $\lambda_{4}$. Therefore, $\lambda_{1}$ and $\lambda_{4}$ are used to transmit down-link (DL) RF data, which usually requires lower OSNR compared to the UL RF data, since we can afford the employment of more sophisticated receivers at the CU. Single-drive MZMs are used to intensity modulate the optical carriers $\lambda_{1}$ and $\lambda_{4}$ with four 64-QAM RF signals that are SCM, as portrayed in Fig 2. Each RF signal has a symbol rate of $20 \mathrm{MSymbols} / \mathrm{s}$ and the resultant SCM signals have center frequencies in the range spanning from $1 \mathrm{GHz}$ to $1.12 \mathrm{GHz}$ for RAU1 and $1.16 \mathrm{GHz}$ to $1.28 \mathrm{GHz}$ for RAU2. Hence the total transmission rate conveyed to each RAU is $480 \mathrm{Mbit} / \mathrm{s}$ ( 4 channels x 20 MSymbols/s x 6 bits per symbol). Our proposed scheme also supports potential RAU sectorization by forwarding each of the four SCM channels to four separate antennas of the sector S1, S2, S3 and S4, as shown in Fig 1. The unmodulated optical carriers $\lambda_{2}$ and $\lambda_{3}$ are coupled together using an Optical Coupler (OC) and attenuated using an Optical Attenuator (OA), before being coupled with the modulated optical carriers $\lambda_{1}$ and $\lambda_{4}$, as seen at the output $\mathrm{OC}$ of the $\mathrm{CU}$ of Fig 2 . The unmodulated carriers $\lambda_{2}$ and $\lambda_{3}$ are attenuated in order to reduce the effect of phase noise due to XPM on the modulated carriers. The phase noise increases upon increasing the average power of the signal, therefore the average power per optical side-band should be kept low. The spectral plot of the resultant optical signal is shown in the inset of Fig 2. It can be observed from the spectral plot that due to the imperfection of OCS and owing to the finite extinction ratio of the AWG based Gaussian filtering, the even-indexed side-bands are still partially present along with the odd-indexed side-bands. The composite optical signal is then transmitted from the CU through $5 \mathrm{~km}$ of Single Mode Fiber (SMF) towards RAU 1 of Fig 1. At RAU 1, the received signal is amplified and Gaussian filtered using the $1 \times 4$ AWG, which has the same parameters as the AWG employed at the transmitter. The EDFA is placed before the AWG so that the Amplified Spontaneous Emission (ASE) noise generated by the EDFA is filtered by the AWG. At RAU 1, the data modulated optical channel $\lambda_{4}$ is dropped. The signal $\lambda_{2}$ is split into two parts using a $3 \mathrm{~dB}$ Optical Splitter (OS), as seen in Fig 2. The lower branch is modulated with the UL RAU1-CU RF data, while the upper branch is coupled with $\lambda_{4}$ and detected by a high-speed Photo-Detector (PD). The PD has a responsivity of $0.8 \mathrm{~A} / \mathrm{W}$, a dark current value of $2 n \mathrm{~A}$ and thermal noise of $10 \times 10^{-12} A / W^{1 / 2}$. At the output of the $\mathrm{PD}$, a $50 \mathrm{GHz}$ mm-wave RF carrier modulated with the RF data is obtained through the process of heterodyning [2]. After the PD, a Gaussian band-pass Electrical Filter (EF) having a 3 $\mathrm{dB}$ bandwidth of $4 \mathrm{GHz}$ and a center frequency of $50 \mathrm{GHz}$ is used to remove the unwanted frequencies from the mm-wave RF signal. At this stage, the amplified output of the EF can be directly transmitted to the MSs from the RAU's antenna, as shown in Fig 2. However, in order to characterize the quality of the received mm-wave signal, it is down-converted to its initial RF frequency using self-mixing [10]. After downconversion, the resultant RF signal having four SCM channels enters the 64-QAM demodulator block seen at the top of Fig 2, where each of the SCM channels is processed separately to obtain Error Vector Magnitude $(\mathrm{EVM})^{1}$ results. It is worth noting here that instead of using $\lambda_{2}$ for heterodyning and UL RAU1-CU data transmission, we can also use the unmodulated signal $\lambda_{3}$ which would generate the mm-wave RF signal at 25 $\mathrm{GHz}$. Hence our method provides two options for the center frequency of the mm-wave RF signal which renders more flexibility in the choice of RF components and services used at the RAU. The UL data signal at RAU1 is also composed of four SCM channels spanning from $1 \mathrm{GHz}$ to $1.06 \mathrm{GHz}$. Each SCM channel is a 64-QAM signal having a symbol rate of $10 \mathrm{MSymbol} / \mathrm{s}$. Hence the total data rate transmitted in the UL direction by a single RAU is $240 \mathrm{Mbits} / \mathrm{s}$. As seen in Fig 2, the ROF signals $\lambda_{1}$ and $\lambda_{3}$ at RAU1 are coupled together and transmitted over $5 \mathrm{~km} \mathrm{SMF}$ towards RAU2. The signal received at RAU2 is amplified and Optically Bandpass Filtered (OBPF) to separate $\lambda_{1}$ and $\lambda_{3}$. The ROF signal $\lambda_{3}$ is then optically split into two paths in Fig 2, one of which is used for heterodyning to generate the mm-wave RF signal, while the other is used for transmitting the UL data received at the RAU2. The mm-wave RF signal is then generated and processed in the same way as at RAU1. The UL SCM RF signal transmitted by RAU2 has center frequencies in the range of $1.16 \mathrm{GHz}$ to $1.22 \mathrm{GHz}$ and has the same data rate as well as modulation format as that of RAU1. After propagating through $5 \mathrm{~km}$ SMF, the ROF signal $\lambda_{3}$ at RAU2 is combined with the UL ROF signal $\lambda_{2}$ of RAU1. The combined signals are received at the $\mathrm{CU}$ after they propagate through another span of $5 \mathrm{~km}$ of SMF. At the CU, the optical signals are filtered and photo-detected to obtain the RF signals. A 64QAM demodulator is used to obtain EVM results of all the RF signals received from each of the RAUs.

\section{Performance Results}

In this section, we present EVM results for both the DL and UL 64-QAM signals. The EVM is plotted against the Signal to

\footnotetext{
${ }^{1}$ The EVM is a measure of the difference between the expected complexvalued voltage of a demodulated symbol and the actual received symbol [11]
} 


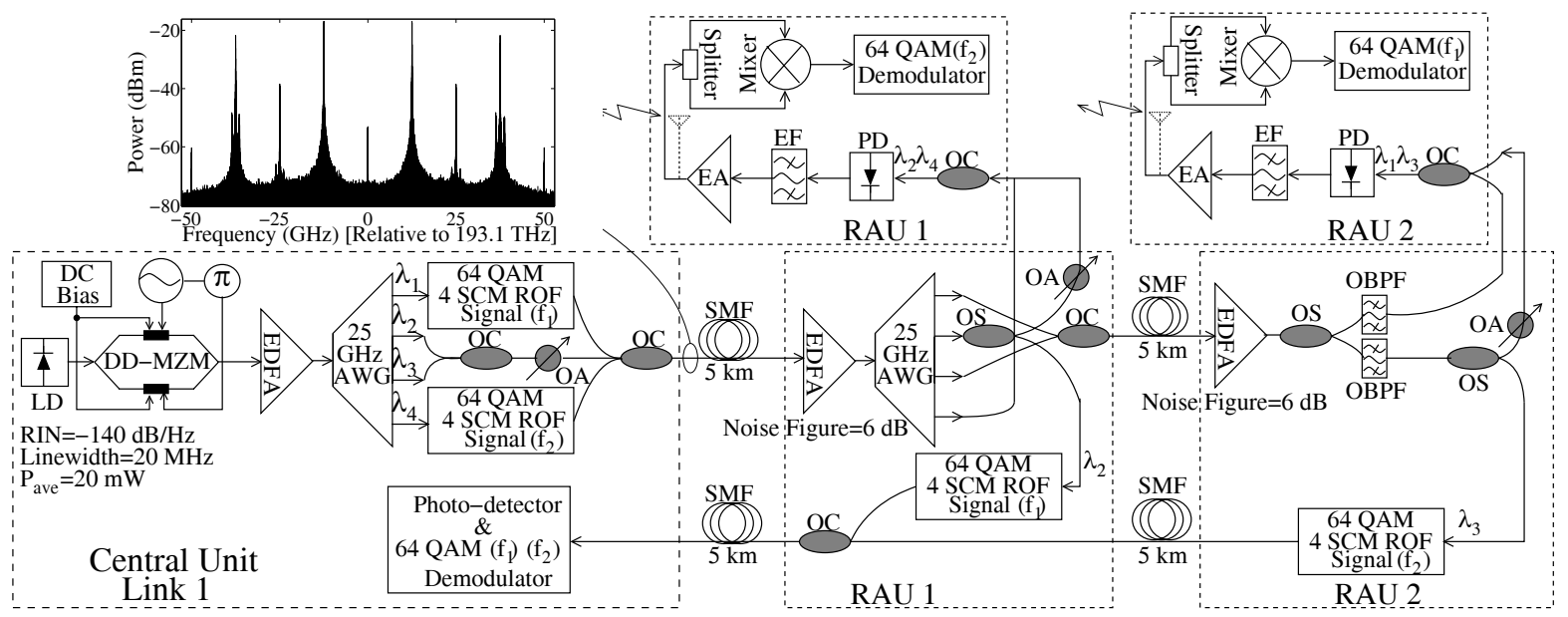

Fig. 2. Schematic of the proposed optical link based virtual MIMO architecture.

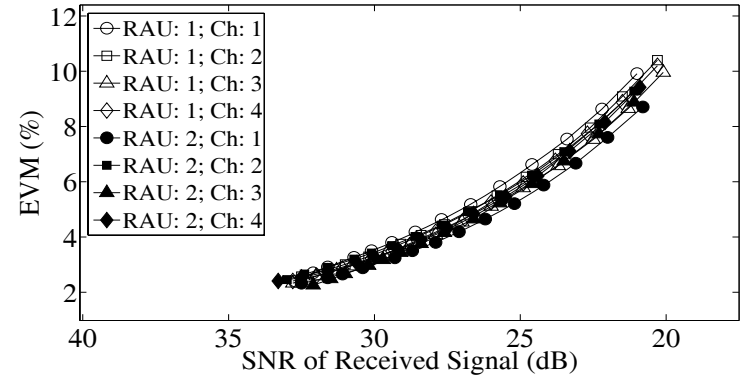

(a)

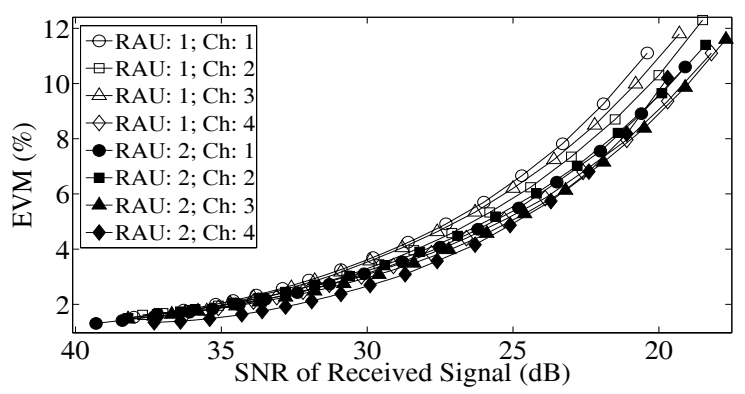

(b)

Fig. 3. EVM vs SNR for (a) CU-RAU DL and (b) RAU-CU UL directions.

Noise Ratio (SNR) of the received RF signal. The value of the SNR is varied by importing in-band optical noise on the ROF channel. Fig 3a and $3 b$ show the results for the DL and UL RF signals, respectively. Since we have used higher-order sidebands for the transmission of DL signals, the maximum SNR achieved for the DL signals is lower than the maximum SNR of UL signals due to the lower OSNR of the DL optical sidebands compared to UL optical side-bands. It can be observed from Fig 3a and $3 \mathrm{~b}$ that all the RF channels of both directions have EVM values that are close to each other. The EVM values achieved for the DL and UL directions are as low as 2.3\% and $1.4 \%$, respectively. These results show that our proposed architecture is suitable for the transmission of high-bit-rate RF signals. In order to provide further insight into the results, we have presented the constellation diagrams in Fig 4 for the third SCM channel of RAU2 received in the DL and UL directions. The SNR values chosen for Fig 4 are set to the maximum

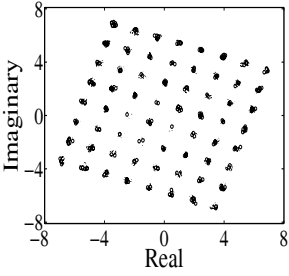

(a) RAU: 2(DL), SNR=32.1dB

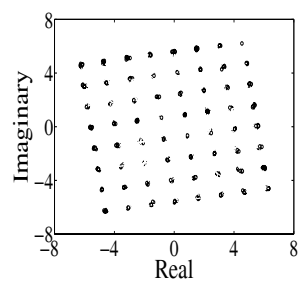

(b) RAU: 2(UL), SNR=38.2dB
Fig. 4. Constellation diagrams for DL and UL signals.

achievable values for channel 3. It can be observed from Fig 4 that the constellation points are confined well within their decision boundaries.

\section{CONCLUSions}

We have demonstrated the transmission of 64-QAM, 4-SCM duplex mm-wave data to two RAUs that are located at $5 \mathrm{~km}$ and $10 \mathrm{~km}$ from the CU in a virtual MIMO architecture. Our technique uses a single laser source to transmit all the data and also allows two different radio frequencies to be generated at the RAU. Good values of EVM are obtained for both the DL and UL signals using components having parameter values close to commercially available components.

\section{REFERENCES}

[1] A. Hekkala, M. Lasanen, I. Harjula, L. C. Vieira, N. J. Gomes, A. Nkansah, S. Bittner, F. Diehm, and V. Kotzsch, "Analysis of and compensation for non-ideal ROF links In DAS," IEEE Wireless Commun., vol. 17, pp. 52-59, June 2010.

[2] T. Kuri and K. Kitayama, "Optical heterodyne detection technique for densely multiplexed millimter-wave-band radio-on-fiber systems," IEEE J. Lightwave Technol., vol. 21, pp. 3167-3179, Dec. 2003.

[3] X. Zhang, B. Liu, J. Yao, K. Wu, and R. Kashyap, "A novel millimeter-wave-band radio-over-fiber system with dense wavelengthdivision multiplexing bus architecture," IEEE J. Lightwave Technol., vol. 54, pp. 929-937, Feb. 2006.

[4] M. F. Huang, J. Yu, Z. Jia, and G. K. Chang, "Simultaneous generation of centralized lightwaves and double/single sideband optical millimeterwave requiring only low-frequency local oscillator signals for radioover-fiber systems," IEEE J. Lightwave Technol., vol. 26, pp. 2653-2662, Aug. 2008.

[5] W. H. Chen and W. I. Way, "Multichannel single-sideband SCM/DWDM transmission systems," IEEE J. Lightwave Technol., vol. 22, pp. 16791693, July 2004. 
[6] J. Yu, G.-K. Chang, Z. Jia, A. Chowdhury, M.-F. Huang, H.-C. Chien, Y.-T. Hsueh, W. Jian, C. Liu, and Z. Dong, "Cost-effective optical millimeter technologies and field demonstrations for very high throughput wireless-over-fiber access systems," IEEE J. Lightwave Technol., vol. 28, pp. 2376-2397, Aug. 2010.

[7] Y.-T. Hsueh, Z. Jia, H.-C. Chien, J. Yu, and G.-K. Chang, "A novel bidirectional $60-\mathrm{GHz}$ radio-over-fiber scheme with multiband signal generation using a single intensity modulator," IEEE Photon. Technol. Lett., vol. 21, pp. 1338-1340, Sep. 2009.

[8] J. James, P. Shen, A. Nkansah, X. Liang, and N. J. Gomes, "Nonlinearity and noise effects in multi-level signal millimeter-wave over fiber transmission using single and dual wavelength modulation," IEEE Trans.
Microw. Theory Tech., vol. 58, pp. 3189-3198, Nov. 2010.

[9] S. Ghafoor and L. Hanzo, "Reduced dispersion duplex DQPSK radio-over-fiber communications using single-laser-based multiple sidebands," in Proc. IEEE International Conference on Communications, pp. 1-5, June 2011.

[10] A. Wiberg, P. Perez-Millen, M. V. Andres, P. A. Andrekson, and P. O. Hedekvist, "Fiber-optic 40-GHz mm-wave link with $2.5-\mathrm{Gb} / \mathrm{s}$ data transmission," IEEE Photon. Technol. Lett., vol. 17, pp. 1938-1940, Sep. 2005.

[11] "IEEE 802.11.a Standardization (ISO/IEC 8802-11:1999/Amd $1: 2000(\mathrm{E})$, 